\title{
SCUBA2 observations of prestellar cores
}

\section{Derek Ward-Thompson and Kate Pattle, on behalf of the JCMT and Herschel-SPIRE Gould Belt Consortia}

\author{
Jeremiah Horrocks Institute, University of Central Lancashire, \\ Preston PR1 2HE, United Kingdom \\ email: dward-thompson@uclan.ac.uk
}

\begin{abstract}
We show data from the SCUBA2 camera on JCMT, of molecular clouds. We focus on starless cores within the clouds. We present data of the Taurus region and show how the environment is affecting some cores' appearance in this region. We compare the SCUBA2 data with Herschel data and discuss the sensitivity of SCUBA2 to surface brightness in the submillimetre. We show how this leads to its ability to pick out the densest cores at a given temperature. Hence SCUBA2 preferentially picks out gravitationally bound pre-stellar cores. We discuss the effects of the magnetic field, and how this lends support to a model for the formation and evolution of cores in filamentary molecular clouds.
\end{abstract}

Keywords. stars: formation - ISM: clouds - ISM: individual objects: L1495 - submillimeter: ISM

\section{Introduction}

Stars form in dense cores in molecular clouds. Exactly how these cores form is still a matter of debate (e.g. André et al., 2014). Cores which do not contain any stars or newlyformed protostars are called starless cores. Those starless cores which are gravitationally bound are known as pre-stellar cores (Ward-Thompson et al., 2007a; Di Francesco et al., 2007). These pre-stellar cores then collapse to form Class 0 protostars (André et al., 1993).

The James Clerk Maxwell Telescope (JCMT) Gould Belt Legacy Survey (GBLS) set out to map all nearby star-forming regions in various spectral lines, and also in the continuum at $850 \mu \mathrm{m}$ with the Sub-mm Common-User Bolometer Array 2 (SCUBA2) camera (Ward-Thompson et al., 2007b). This data-set can be used in comparison with the Herschel Gould Belt Survey (HGBS) at wavelengths from 70 to $500 \mu \mathrm{m}$ (André et al., 2010; Pattle et al., 2015).

Cores are a significant stage in star formation. Recent work by the HGBS and the JCMT GBLS has led to new insight into core formation (André et al., 2014). One of the goals of the GBLS was to catalogue and map all of the pre-stellar cores within about $0.5 \mathrm{kpc}$ (Ward-Thompson et al., 2007b). The mass function of cores can then be modelled onto the IMF of stars (Goodwin et al., 2008).

\section{Taurus}

Figure 1 shows the L1495 region in Taurus (Ward-Thompson et al., 2016). Five Herschel wavebands and one waveband from SCUBA2 are shown. A number of cores and filamentary structures can be seen. In particular a triangle-shaped arrangement of filaments can be seen just below centre of each image. The ubiquity of filaments is a feature seen in almost all of the Herschel data of star-forming regions (André et al., 2010). 


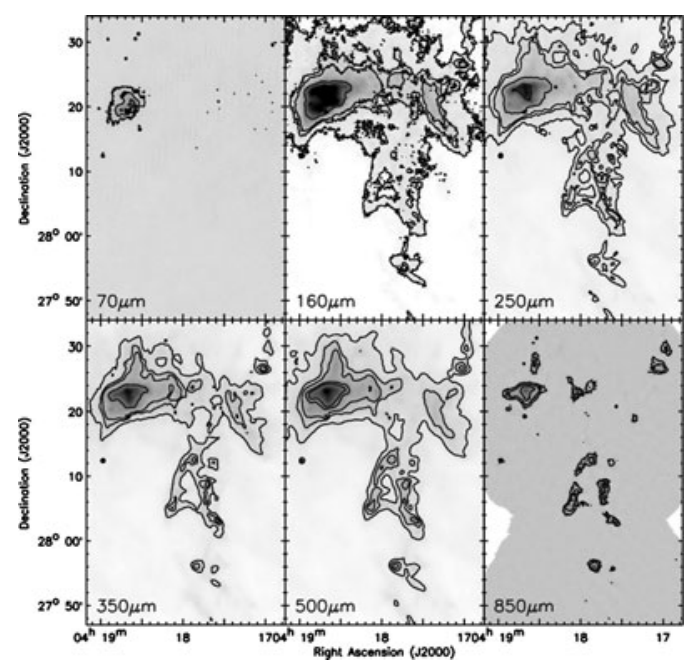

Figure 1. Herschel and SCUBA2 images of the L1495 molecular cloud in Taurus. Top row, left to right: 60,170 and $250 \mu \mathrm{m}$ respectively. Lower row, left to right: 350 and $500 \mu \mathrm{m}$ (all from Herschel) and $850 \mu \mathrm{m}$ (from SCUBA2) respectively. Note how SCUBA2 only detects some of the cores seen at the other wavelengths by Herschel (Ward-Thompson et al., 2016).

The bright region towards the upper part of the image that is seen at most wavebands is the L1495A core, and is exactly coincident with L1495A-S. This lies at the head of a large, filamentary structure that is seen clearly in the Herschel data of the Taurus region (Marsh et al., 2016). We measured a temperature gradient across this core, with the hotter material lying to the south (Ward-Thompson et al., 2016).

There is a bright star, slightly to the south of L1495A-S, which is known as V892 Tau (IRAS04155+2812). This is a Herbig Ae/Be star, and it is clearly heating L1495A-S, which is otherwise starless, and causing the temperature gradient across the core. So we see that environment is affecting the appearance of some cores in the far-infrared and sub-mm. Similar effects were seen in cores in Cepheus (Nutter et al., 2009; Pattle et al., 2016). Note that only some of the cores visible in the Herschel images are seen in the SCUBA2 image. For example, the filament to the upper right in the Herschel images appears to Herschel as no different from the other filaments, but it is almost invisible in the SCUBA2 image.

Figure 2 shows a plot of density versus temperature for the starless cores in the L1495 region. The cores detected by SCUBA2 are indicated by black crosses. Those that are detected by Herschel are indicated by green crosses. The cores detected by SCUBA2 all lie in the upper part of this plot (Ward-Thompson et al., 2016). However, note that both sets of cores occupy a similar range of temperatures.

Figure 3 shows a plot of mass versus deconvolved full-width at half-maximum (FWHM) size for the starless cores in the L1495 region. The cores detected by SCUBA2 are indicated by black points. Those that are detected by Herschel are indicated by green points. The cores detected by SCUBA2 all lie in the upper part of this plot (Ward-Thompson et al., 2016). However, both set of cores occupy a similar range of sizes.

Figures 2 and 3 taken together would appear to indicate that SCUBA2 is only sensitive to the densest cores, or to the most massive cores for a given size, whereas Herschel sees more of the cores (Ward-Thompson et al., 2016). This can be understood in terms of a surface brightness effect in the SCUBA2 data. For a given, relatively narrow, range of temperatures, such as is observed in starless cores, SCUBA2 surface brightness traces 


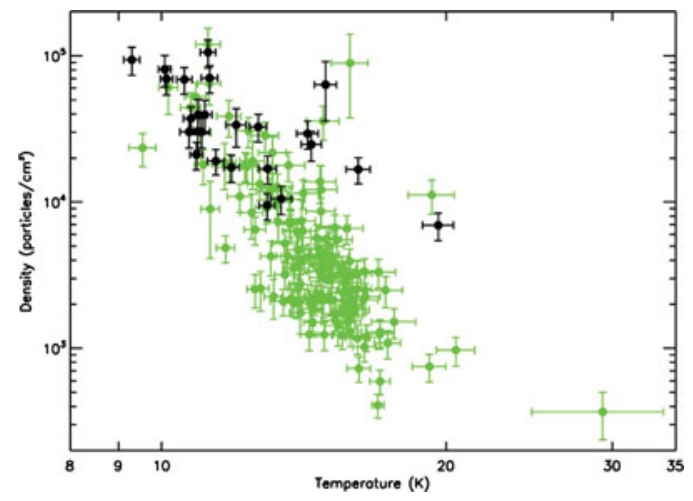

Figure 2. Density plotted against temperature for Herschel (grey crosses) and SCUBA2 (black crosses) data of starless cores. Note how both data-sets span a similar range of temperatures, but that the SCUBA2 cores only occupy the higher density parts of the plot (Ward-Thompson et al., 2016).

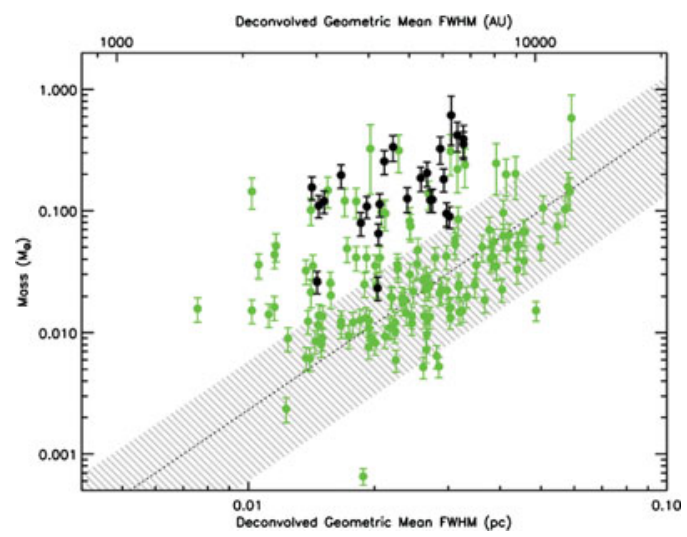

Figure 3. Mass plotted against deconvolved FWHM for Herschel (grey crosses) and SCUBA2 (black crosses) data of starless cores. Note how both data-sets span a similar range of FWHM size, but that the SCUBA2 cores only occupy the higher mass parts of the plot (Ward-Thompson et al., 2016).

column density. Furthermore, for normally non-elongated structures, such as starless cores, column density effectively traces volume density. Hence the surface brightness of a starless core seen in a SCUBA2 image traces the density of that core. All of this implies that SCUBA2 will preferentially detect the densest cores - i.e. those closest to gravitational collapse - pre-stellar cores (Ward-Thompson et al., 2016).

\section{Magnetic fields}

The magnetic field direction (in the plane of the sky) in a molecular cloud can be inferred from sub-mm dust continuum polarization measurements. Figure 4 shows results from the Ballon-borne Large Aperture Sub-mm Telescope Polarimeter (BLASTPol) of a filamentary structure in the Lupus molecular cloud (Matthews et al., 2014). The curved line traces the axis of the filament. The vectors trace the direction of the magnetic field inferred from the polarization measurements. Note how the B-field lies largely perpendicular to the direction of the filament axis (Matthews et al., 2014).

Observations such as these have led to a new model being proposed for core formation (André et al., 2014). In this new scenario, material is funnelled onto filaments by the 


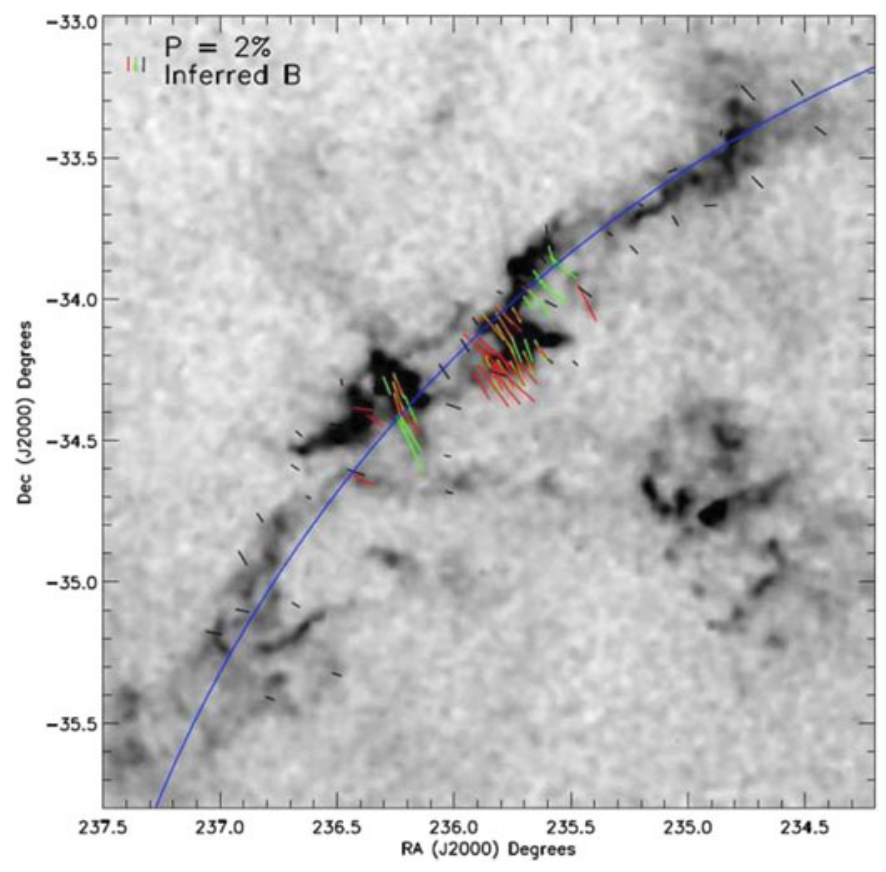

Figure 4. BLASTPol B-field vectors superposed on a sub-mm image of the Lupus molecular cloud. The vectors trace the direction of the magnetic field. The curved line traces the axis of the filament. Note how the B-field lies largely perpendicular to the direction of the filament axis (Matthews et al., 2014). (A color version of this figure is available online.)

magnetic field (Palmeirim et al., 2013). Dense parts of the filaments form cores by accreting further matter along the filaments. The filaments accrete mass until they exceed the critical mass per unit length against gravitational instability (Kawachi \& Hanawa 1998). At this point they collapse under self-gravity to form proto-stellar cores. Herschel observed that roughly two-thirds of cores form in this manner (Könyves et al., 2015).

\section{References}

André, P., Ward-Thompson, D., \& Barsony, M. 1993, ApJ 406122

André, P., Men'shchikov, A., Bontemps, S., et al. 2010, A $\& A 518102$

André, P., Di Francesco, J., Ward-Thompson, D., et al. 2014, Protostars \& Planets VI p. 27

Di Francesco, J., Evans, N. J. II, Caselli, P., et al. 2007, Protostars \& Planets V p. 17

Goodwin, S. P., Nutter, D., Kroupa, P., et al. 2008, A\& $A 477823$

Kawachi, T. \& Hanawa, T., 1998, PASJ 50577

Könyves, V., André, P., Mensh'chikov A., et al. 2015, A\&̊A, 584, A91

Marsh, K. M., Kirk, J. M., André, P., et al. 2016, MNRAS submitted

Matthews, T. G., Ade, P. A. R., Angilè, F. E., et al. 2014, ApJ 784116

Nutter, D., Stamatellos, D., Ward-Thompson, D. 2009 MNRAS 3961851

Palmeirim, P., André, P. Kirk, J. M., Ward-Thompson, D., et al. 2013, A\& $A 550$ A38

Pattle, K., Ward-Thompson, D., Kirk, J. M., White, G. J. et al. 2015, MNRAS 4501094

Pattle, K., Ward-Thompson, D., Keown, J., et al. 2016 MNRAS in prep.

Ward-Thompson, D., André, P., Crutcher, R., et al. 2007, Protostars \& Planets V p.33

Ward-Thompson, D., Di Francesco, J., Hatchell, J., et al. 2007, PASP 119855

Ward-Thompson, D., Pattle, K., Kirk, J. M. et al. 2016, MNRAS in prep. 